\section{$\underset{\substack{\text { hommes } \\ \text { \& migrations }}}{ }$}

\section{Hommes \& migrations}

Revue française de référence sur les dynamiques

migratoires

\section{8 | 2014}

Les Paris des migrants

\title{
Les étudiants étrangers à Paris en 1900
}

\section{Pierre Moulinier}

\section{OpenEdition \\ Journals}

Édition électronique

URL : http://journals.openedition.org/hommesmigrations/3011

DOI : 10.4000/hommesmigrations.3011

ISSN : 2262-3353

\section{Éditeur}

Musée national de l'histoire de l'immigration

Édition imprimée

Date de publication : 1 octobre 2014

Pagination : 119-127

ISBN : 978-2-919040-29-2

ISSN : $1142-852 X$

\section{Référence électronique}

Pierre Moulinier, «Les étudiants étrangers à Paris en 1900 », Hommes \& migrations [En ligne], 1308 | 2014, mis en ligne le 01 octobre 2017, consulté le 30 avril 2019. URL : http:// journals.openedition.org/hommesmigrations/3011; DOI : 10.4000/hommesmigrations.3011 


\title{
LES ÉTUDIANTS ÉTRANGERS À PARIS EN 1900
}

par PIERRE MOULINIER, Groupe d'études et de recherche sur les mouvements étudiants.

\author{
À la veille de la Première Guerre mondiale, dans les universités \\ parisiennes, près de 1 étudiant sur 6 est étranger. Parmi \\ les nationalités représentées, on compte une part importante \\ de Russes, de Turcs, de Roumains ou d'Allemands venus acquérir \\ une formation universitaire de qualité. Le Paris de la Belle \\ Époque étend son rayonnement culturel sur le reste de l'Europe \\ et au-delà. Tout en accueillant favorablement ces migrants pour \\ études, les autorités françaises tentent toutefois d'en limiter \\ l'installation.
}

En 1900, Paris est la capitale intellectuelle, artistique et littéraire du monde. Signe de cette attirance, à la fin du XIX siècle, les cinq facultés parisiennes (droit, médecine, lettres, sciences et pharmacie) accueillent de nombreux étudiants et étudiantes étrangers venus de tous les continents ${ }^{1}$. En dehors des séductions multiples de la capitale, une des raisons de son attirance est que la France se distingue des autres nations universitaires (Allemagne, Grande-Bretagne ou Autriche) par une politique d'accueil très libérale d'étudiants étrangers, et cela dès le début du XIX ${ }^{e}$ siècle. En 1840, dans une circulaire adressée aux recteurs, le ministre de l'Instruction publique Victor Cousin écrit: "La noble hospitalité que la France a toujours offerte aux étrangers, et l'antique réputation de ses grandes écoles scientifiques, juridiques et littéraires ont sans cesse attiré un grand nombre de jeunes gens de diverses contrées qui viennent puiser parmi nous les connaissances qu'ils apporteront ensuite dans leur patrie. Il convient de favoriser ces habitudes dans l'intérêt de la gloire du nom français, et aussi dans l'intérêt de nos établissements universitaires ${ }^{2}$." Mais cette raison n'est pas suffisante : la poussée étrangère vers la France s'explique surtout par des motivations très hétérogènes de la part des jeunes et des 
familles, différentes d'un pays à l'autre, en fonction de contextes économiques, politiques et culturels nationaux variables.

\section{Paris, un pôle d'attraction au tournant du siècle}

Entre 1880 et 1914, le nombre d'étudiants dans l'ensemble des facultés françaises, français et étrangers, passe de 12000 à 42 000. Dans le même temps, la population des facultés parisiennes qui ne regroupaient que 4000 individus en 1865 s'élève à 6700 en 1882, dépasse les 10000 en 1892 et atteint le chiffre record de 19001 en 1910. Dès 1910, une diminution s'observe, provoquée en partie par la crise économique et, dès 1912, par l'effet des guerres balkaniques sur les effectifs étrangers. La population étudiante de Paris se stabilise alors autour de 17500 , avant la chute brutale due à la guerre (4 257 en 1914, 6025 en 1916). La remontée s'opère en 1919, où l'on retrouve le chiffre des années précédant le conflit mondial (17 761).

À partir des années 1880, on constate en France, comme dans d'autres pays occidentaux (Allemagne, Suisse, Autriche-Hongrie, Belgique, Italie, GrandeBretagne), une importante augmentation des étrangers. La croissance de leurs effectifs durera jusque dans les années 1930 : ils ne constituaient que $5,6 \%$ de la population étudiante totale en France en 1890, ils en représentent près de $14 \%$ en moyenne en 1910-1914, 15 \% en 1910. À Paris, de 1882 à 1914, résultat de l'effort des pouvoirs publics en vue d'attirer la clientèle étrangère dans les facultés, le nombre des étrangers des deux sexes inscrits dans les facultés parisiennes est multiplié par plus de quatre, soit plus que l'accroissement de la population des étudiants français, qui ne fait que doubler. Ce chiffre se réduit à une moyenne de 1429 par an pendant la guerre, soit moitié moins, et remonte à 3564 en 1920. Les premières étudiantes entrent dans les facultés à partir de 1870. À Paris, les effectifs féminins connaissent une hausse de 1889-1890 à 1895-1896 (de 195 à 522), diminuent jusqu'au tournant du siècle autour de 400, pour croître ensuite rapidement à 2197 en 1913-1914. De 2 \% des effectifs en 18861887, on passe à $12,7 \%$ avant la Grande Guerre. Quant aux étrangères, elles passent entre 1890 et 1909 de 139 à 1284, soit près de dix fois plus, et à plus de 1950 en 1914 ; parmi les étudiantes, les étrangères l'emportent sur les Françaises jusqu'à la Grande Guerre.

Les étrangers se répartissent différemment entre les facultés parisiennes : entre 1890 et 1914, ils passent de $5,7 \%$ à $12,1 \%$ en droit, de $1,5 \%$ à 2,8 \% en pharmacie, de 7,8 \% à 34,9\% en lettres, de $10,4 \%$ à $24 \%$ en sciences ; et si leur proportion s'élève à 18,5 \% dans les années 1890-1894 en médecine, ce pourcentage diminue au début du $\mathrm{XX}^{\mathrm{e}}$ siècle en raison de violentes campagnes contre l'excès de diplômés en France (licenciés en droit ou docteurs en médecine), campagnes qui concernent aussi, bien entendu, l'accès des étrangers aux carrières médicales. Dans les années 1900-1904, la proportion d'étudiants en médecine étrangers n'est que de 9,9\%, mais elle remonte à 20,8 \% à la veille de la Grande Guerre. Enfin, la plus grande partie des étrangères sont inscrites à la faculté de médecine ou à la faculté des lettres.

\section{La provenance des étudiants étrangers}

S'agissant des Européens ${ }^{3}$, deux pays l'emportent de loin sur les autres au tournant du siècle : les Russes (dominant en médecine et en sciences, et parmi lesquels on compte de nombreuses femmes) ${ }^{4}$ et les Roumains (médecine, droit et sciences), loin devant les pays balkaniques (Grèce, Serbie, Bulgarie) et les pays voisins de la France (Angleterre, Belgique, PaysBas, Suisse, Allemagne). On remarque toutefois que les ressortissants des pays occidentaux riches d'une vieille culture et d'équipements universitaires 
// Effectifs des étudiants et des étudiantes à Paris (1890-1913)

\begin{tabular}{|c|c|c|c|c|}
\hline & Nombre d'étudiants & Dont étudiantes & Nombre d'étrangers & Dont étrangères \\
\hline $1889-1890$ & 8653 & 195 & 1078 & 139 \\
\hline 1890-1891 & 9215 & 210 & 896 & 111 \\
\hline 1891-1892 & 9925 & 244 & 1049 & 132 \\
\hline $1892-1893$ & 10110 & 247 & 1054 & 127 \\
\hline 1893-1894 & 10577 & 390 & 1193 & 175 \\
\hline 1894-1895 & 10951 & 453 & 1189 & 210 \\
\hline 1895-1896 & 11170 & 522 & 1258 & 234 \\
\hline 1896-1897 & 10545 & 469 & 1067 & 201 \\
\hline $1897-1898$ & 12047 & 400 & 1129 & 165 \\
\hline 1898-1899 & 11829 & 325 & 992 & 129 \\
\hline $1899-1900$ & 12171 & 402 & 1118 & 197 \\
\hline 1900-1901 & 12289 & 401 & 1162 & 205 \\
\hline 1901-1902 & 13432 & 511 & 1188 & 266 \\
\hline $1902-1903$ & 12574 & 612 & 1241 & 336 \\
\hline 1903-1904 & 12985 & 702 & 1312 & 401 \\
\hline 1904-1905 & 13431 & 935 & 1560 & 544 \\
\hline 1905-1906 & 13908 & 1231 & 1911 & 710 \\
\hline 1906-1907 & 16022 & 1341 & 2205 & 856 \\
\hline 1907-1908 & 17303 & 1773 & 2808 & 1133 \\
\hline 1908-1909 & 17903 & 2010 & 3072 & 1205 \\
\hline 1909-1910 & 19001 & 2211 & 3292 & 1284 \\
\hline $1910-1911$ & 17711 & 2121 & 3217 & 1168 \\
\hline $1911-1912$ & 17802 & 2190 & 3384 & 1174 \\
\hline $1912-1913$ & 17545 & 2068 & 3223 & 1090 \\
\hline 1913-1914 & 17246 & 2197 & 3242 & 1077 \\
\hline
\end{tabular}

Source : Edmée Charrier, L'Évolution intellectuelle féminine, Paris, Mechelink, 1931.

prestigieux apprécient les études françaises dispensées par la Sorbonne et s'inscrivent volontiers à la faculté des lettres. On constate également le développement de la colonie égyptienne (de 2,5 \% à $7 \%$ en 1900), essentiellement en droit. Il faut surtout noter la montée en puissance des sujets de l'Empire ottoman, en particulier en droit et en médecine, dont le poids passe entre 1890 et 1899 de $10 \%$ à 13 ou $14 \%$, pour décroître ensuite jusqu'à $4,2 \%$ en 1909 et remonter, selon l'année, entre $7 \%$ et près de $9 \%$. Enfin, les originaires des divers pays de l'Amérique latine et des Caraïbes sont également 
Répartition des étudiants étrangers à Paris par nationalités et facultés en 1904

\begin{tabular}{|c|c|c|c|c|c|c|c|}
\hline & Droit & Médecine & Sciences & Lettres & Pharmacie & Total & $\%$ \\
\hline Allemagne & 5 & 4 & 7 & 94 & - & 110 & 8,1 \\
\hline Autriche-Hongrie & 4 & 4 & 7 & 37 & - & 52 & 3,8 \\
\hline Benelux & 9 & 7 & 9 & 14 & - & 39 & 2,9 \\
\hline Bulgarie & 17 & - & - & 1 & - & 18 & 1,3 \\
\hline Espagne-Portugal & - & 8 & 3 & - & - & 11 & 0,8 \\
\hline Grèce & 9 & 22 & 3 & 2 & - & 36 & 2,6 \\
\hline Îles britanniques & 10 & 7 & - & - & 1 & 18 & 1,3 \\
\hline Italie & 5 & 5 & 2 & 3 & 1 & 16 & 1,2 \\
\hline Pays nordiques & - & - & 1 & 7 & - & 8 & 0,6 \\
\hline Roumanie & 81 & 53 & 27 & 6 & 1 & 168 & 12,4 \\
\hline Russie & 45 & 132 & 153 & 135 & 1 & 466 & 34,4 \\
\hline Serbie & 18 & 2 & 3 & 2 & - & 25 & 1,8 \\
\hline Suisse & 8 & 14 & 15 & 8 & 1 & 46 & 3,4 \\
\hline Turquie & 20 & 66 & 13 & 2 & 7 & 108 & 8,0 \\
\hline États-Unis-Canada & 1 & 2 & 5 & 54 & - & 62 & 4,6 \\
\hline Amérique lat., Caraïbes & 17 & 41 & 6 & 4 & 6 & 74 & 5,4 \\
\hline Égypte & 68 & 5 & 1 & - & 1 & 75 & 5,6 \\
\hline Autres Afrique & 1 & 8 & - & - & 1 & 10 & 0,7 \\
\hline Perse & 1 & 4 & - & - & 1 & 6 & 0,4 \\
\hline Japon-Chine & 3 & - & 1 & 1 & - & 5 & 0,4 \\
\hline Autres Asie-Océanie & - & 1 & - & - & - & 1 & - \\
\hline Total & 322 & 385 & 256 & 371 & 21 & 1355 & \\
\hline$\%$ & 23,8 & 28,4 & 18,9 & 27,4 & 1,5 & & $100 \%$ \\
\hline
\end{tabular}

Source : Rapport du recteur de l'Académie de Paris pour 1904.

très présents en droit et en médecine. L’Amérique du Nord (Canada et surtout États-Unis), encore très représentée à la faculté de médecine en 1890 et 1891, délaisse cette discipline à la fin du siècle et, après 1898, n'envoie plus que de 3 à 7 représentants. Les États-Unis n'envoient à la faculté de droit qu'une poignée d'étudiants, fréquentent peu la faculté des sciences, mais choisissent volontiers la faculté des lettres, où ils passent d'une dizaine en 1898 à 91 en 1909 et à 77 en 1913.
On note, à la Belle Époque, un véritable afflux d'étudiants et d'étudiantes russes ${ }^{5}$. La Russie figure quasiment en première place parmi les principales nationalités présentes dans les facultés parisiennes. En droit : la Roumanie, l'Égypte, la Turquie, la Serbie, la Grèce, la Bulgarie ; en médecine : la Russie, la Roumanie, la Suisse, l'Allemagne, la Grèce, la Turquie ; en sciences : la Russie, la Roumanie, la Turquie ; en lettres : la Russie, la Grande-Bretagne, l'Allemagne, l'Autriche-Hongrie, les États-Unis ; en 
pharmacie : la Russie, la Turquie. Les raisons de cette présence russe sont autant la restriction des effectifs dans les universités russes, qui sont réservées aux élèves des gymnases classiques et privilégient les couches supérieures de la société impériale, en particulier la noblesse, que les mesures antijuives instituées sous la forme du numerus clausus dans les universités en 1887. C'est la raison pour laquelle les juifs sont nombreux dans la communauté russe et représentent plus de la moitié des docteurs en médecine russes reçus à Paris. Ajoutons pour les Polonais placés sous la botte du tsar les mesures de "russisation" des universités polonaises. La pratique du français par les élites est également une raison de se rendre à Paris. Du côté des femmes, d'autres motifs expliquent leur émigration. À ce que le professeur Victor Karady ${ }^{6}$ appelle la "surscolarisation", c'est-à-dire un bon niveau d'études secondaires sans débouchés intéressants sur l'enseignement supérieur, s'ajoute pour les juives et pour les Polonaises la persécution qui les frappe. La Russie est l'une des premières nations à ouvrir des cours supérieurs féminins, notamment de médecine, et c'est leur fermeture en 1885-1886 qui provoque la fuite des étudiantes vers l'ouest, notamment en Suisse et en France?

\section{Les raisons de l'attrait de la France}

En dépit de mouvements d'hostilité qui sont à la fin du siècle surtout le propre des étudiants français en médecine, des médecins praticiens et des syndicats médicaux naissants, force est de constater que, tout au long du XIX siècle, la France se distingue dans le concert européen par l'accueil très libéral réservé aux étudiants étrangers. Les universitaires et les hommes politiques de la III République s'accordent sur des mesures d'ouverture qui ne peuvent, selon eux, que renforcer le prestige mondial de la France et lui gagner des soutiens extérieurs. Les outils de cette politique sont en particulier la création d'établissements culturels et éducatifs à l'étranger (instauration de collèges et lycées français ou de facultés professionnelles, notamment en Grèce, en Roumanie, en Égypte et en Turquie, réseau de l'Alliance française fondé en 1883), une propagande efficace, l'octroi de bourses à certains étudiants étrangers (des pays balkaniques en particulier), la mise en place de comités de patronage et de centres d'information dans les villes universitaires au profit des étrangers, des mesures destinées à faciliter leurs études en France (équivalences et dispenses d'examen, cours de français, diplômes adaptés, tels les doctorats d'université qui n'ont pas de valeur professionnelle dans l'Hexagone, etc.) $)^{8}$. Cette politique d'ouverture fait que la France vient en tête dans le nombre d'étudiants étrangers accueillis.

Ces mesures favorables ne peuvent que satisfaire la demande de nombreux pays et des familles des classes aisées. Quelles sont en effet les motivations de ces voyageurs intellectuels ? On peut distinguer plusieurs sortes d'étudiants migrants ${ }^{9}$.

Il y a ceux en quête d'un diplôme occidental. Les migrations étu-
Tout au long du XIXe siècle, la France se distingue dans le concert européen par l'accueil très libéral réservé aux étudiants étrangers. du XIX ${ }^{e}$ siècle et du premier XX $X^{e}$ siècle s'insèrent dans une internationalisation du marché universitaire qui met en compétition trois "modèles" dominants : le français, l'allemand et le britannique, en lutte pour capter la demande des pays sous-développés en matière de formation de cadres nationaux et désireux de moderniser leur appareil universitaire, politique, administratif, scientifique, sanitaire et social. Il en résulte une domination culturelle de l'Ouest européen sur l'Orient européen et méditerranéen. Cette situation est parti- 
culièrement celle de la Roumanie, de la Serbie, de l'Égypte, de la Bulgarie, de la Grèce, de l'Empire ottoman, qui envoient des boursiers à Paris en vue d'obtenir un doctorat en droit ou en médecine et même exigent l'obtention d'un diplôme français pour exercer dans le pays émetteur, comme avant la Grande Guerre en Roumanie. Les réfugiés constituent une autre catégorie d'étrangers regroupant les "exclus des marchés universitaires nationaux" : les minorités linguistiques ou ethniques, les juifs et les femmes. On a vu l'importance des phénomènes d'exclusion dans la Russie du second XIX siècle, mais d'autres communautés étrangères peuvent trouver à Paris un havre salutaire.

Un troisième groupe, les "cosmopolites" et les francophiles, rassemble les étudiants qui s'expatrient pour éviter l'assimilation à une élite locale jugée rétrograde, ainsi que ceux qui éprouvent une forte attirance pour la France. On connaît à ce titre la francophilie des élites rou-
Les étudiants étrangers

du XIXe siècle doivent

présenter une équivalence

du baccalauréat exigé

des Français ou une dispense de titre secondaire maines, bulgares, russes ou serbes.

Enfin, il y a les "touristes" culturels ou politiques. À côté des boursiers, des jeunes sont envoyés par leurs familles pour acquérir un titre prestigieux, parfois déjà obtenu par leur père, ou parce qu'ils souhaitent mener des "études d'agrément", voire faire du tourisme culturel. C'est le cas des fils de boyards roumains ou russes, des rejetons de riches familles égyptiennes. Certains sont animés par des idéaux sociaux ou politiques et quelquesuns d'entre eux radicaliseront leurs positions politiques au contact des valeurs républicaines ou révolutionnaires françaises. Ainsi, des jeunes Roumains de 1848 qui porteront la révolution dans leur pays ou y provoqueront des réformes sociales, telles que l'abolition du servage. Le Quartier latin devient ainsi une école de nationalisme et un séminaire pour futurs cadres politiques.

\section{Le choix des études}

Comme aujourd'hui, pour accéder aux études supérieures en France, il faut être en possession d'un baccalauréat ${ }^{10}$. Les étudiants étrangers du XIX siècle doivent présenter une équivalence du baccalauréat exigé des Français ou une dispense de titre secondaire. La nature des documents demandés est précise : l'étudiant étranger doit fournir au rectorat un certificat du ministre de l'Éducation de son pays, l'original des diplômes dont il sollicite l'équivalence avec éventuellement une traduction certifiée conforme. Parfois, le visa d'un consul de France, comme en Russie, est nécessaire. Les diplômes recherchés sont, à la faculté de droit : le baccalauréat, la licence et le doctorat ; à la faculté de médecine : le doctorat ; à l'École supérieure de pharmacie : le diplôme de pharmacien de $1^{\text {re }}$ classe ou de $2^{\mathrm{e}}$ classe ; dans les facultés des lettres et des sciences : la licence et le doctorat. Ce sont des diplômes d'État qui seront, à la fin du siècle, de moins en moins accessibles aux étrangers. Dans les années 1890, sur fond de xénophobie et d'antisémitisme, des manifestations de médecins et d'étudiants en médecine sont organisées contre la concurrence que feraient peser les docteurs étrangers sur la pratique des médecins français. Le slogan de l'époque est la "pléthore médicale", c'est-àdire l'excès de médecins exerçant en France et le trop grand nombre de doctorats décernés chaque année. Comme aucun texte n'interdit aux docteurs étrangers pourvus du diplôme français d'exercer la médecine en France, les manifestants demandent que les étrangers ne puissent plus obtenir le doctorat d'État. Il en résulte la création en 1897 des "diplômes d'université", décernés par les universités et non par l'État, qui permettent aux étrangers de quitter la France avec un titre analogue au diplôme d'État, mais qui leur interdit de tra- 
vailler en France. En 1900, le nombre d'étrangers reçus docteurs d'État en médecine commence à diminuer.

\section{La vie quotidienne à Paris}

En dehors des périodes de vacances et sauf imprévus, les étudiants étrangers effectuent des séjours de trois à huit ans dans la capitale - plutôt trois ans en droit, de cinq à huit ans en médecine et plus de six ans en pharmacie. Comme je l'ai montré dans mon ouvrage sur les étudiants parisiens au XIX ${ }^{e}$ siècle, la fin du siècle est caractérisée par une large attention des universitaires au mode de vie des étudiants ${ }^{11}$. En réponse à une question posée dans le Journal des débats du $1^{\text {er }}$ février 1891 sur les conditions de vie des étudiants étrangers à Paris, le secrétaire général du comité de patronage des étudiants étrangers Paul Melon répond qu'il s'en est lui-même inquiété, car l'étranger ne doit pas "partir de chez soi à l'étourdie, et le temps où l'on croyait à la bohème laborieuse est passé". Selon cet auteur, on peut estimer ainsi le budget d'un étudiant étranger dans la capitale : "Voici : la chambre coûte par mois 35 ou 40 francs en moyenne; il faut ajouter 5 francs pour le service; la blanchisserie revient à 10 francs environ; le chauffage et l'éclairage (pendant les mois d'hiver) à 14 francs; la nourriture varie: on déjeune à 22 sous et on dîne à 24 ; les Russes (s'ils sont princes) peuvent aller jusqu'à 2 francs pour le déjeuner et 2,50 pour le dîner; il y a des pensions à 90, 100 et 120 francs par mois. Beaucoup d'autres dépenses ne peuvent se compter qu'à l'année : vêtements, chaussures, chapeaux, linge, livres, cotisation à l'Association des étudiants, où le toit et la lumière sont offerts, avec des conférences qu'il est possible d'entendre et possible d'éviter. Bref, avec 250 francs par mois, un étudiant peut mener presque la grande vie. Et, en instruisant de plus ignorants que lui (il en trouvera toujours), il peut gagner lui-même au moins la moitié de cette somme ${ }^{12 .}$."
Les ressources financières des étudiants étrangers proviennent en majorité de leurs parents, à moins qu'ils ne bénéficient d'une bourse de leur gouvernement. Cette situation crée une forte disparité entre les étudiants qui étalent leur richesse et ceux, pauvres, qui se recrutent en majorité chez les Russes. Beaucoup de ces derniers ont des moyens limités, ce qui explique qu'ils se rassemblent au Quartier latin. Le poète et journaliste Fernand Divoire décrit un style de vie misérable: "Pauvres et sales pour la plupart, ils vivent avec les 60 francs par mois que leur envoient leurs familles. Ils couchent où ils peuvent, chez les uns et chez les autres, à plusieurs dans de petites chambres où ils boivent du thé concentré étendu d'eau chaude, où ils parlent de la justice, mais où ils ne font pas de bombes ${ }^{13}$." Les étrangers, comme les provinciaux, sont logés au Quartier latin, c'est-à-dire dans les $\mathrm{V}^{\mathrm{e}}$ et $\mathrm{VI}^{\mathrm{e}}$ arrondissements actuels ${ }^{14}$.
En dehors des périodes de vacances et sauf imprévus, les étudiants étrangers effectuent des séjours de trois à huit ans dans la capitale - plutôt trois ans en droit, de cinq à huit ans en médecine et plus de six ans en pharmacie. quelques foyers pour les étudiantes, notamment étrangères, la politique du logement étudiant ne débute à Paris que dans l'entre-deux-guerres avec la Cité universitaire, qui voit le jour en 1925. Avant la Grande Guerre, les étudiants étrangers qui ne sont pas logés chez des parents ou compatriotes sont assujettis aux mêmes obligations que leurs camarades français venus de leur province : la pension ou l'hôtel, à choisir selon les ressources.

\section{Des étudiants destinés à repartir dans leur pays d'origine}

La France, pays ouvert aux études des étrangers, est plutôt réticente à l'immigration des diplômés étrangers, en particulier des médecins, même 


\section{LA COLONIE RUSSE DES GOBELINS}

Dans les décennies qui entourent l'an 1900, les étudiants et les étudiantes russes se regroupent en grand nombre dans un quartier sis autour de la place des Gobelins, dans une zone géographique qui a le mérite de rester proche du Quartier latin et des facultés, et d'offrir des logements à loyers modérés à une communauté étrangère célèbre pour la modestie de ses ressources et de ses besoins. Pour les nombreux étudiants en médecine qu'elle comporte, le quartier a en outre le mérite de se situer à proximité des hôpitaux où ont lieu certains cours et travaux pratiques (Cochin, Port-Royal, le Val-de-Grâce) et près d'amphithéâtres de dissection, tels celui dit de Clamart, rue du Fer-à-Moulin. Ce quartier a retenu l'attention des contemporains ${ }^{1}$ - et de la police - en raison de l'importance numérique de cette colonie étudiante et des ferments révolutionnaires qu'elle aurait abrités.

Au début du XXe siècle, Paris compterait 25000 Russes, dont 12000 adultes ; les trois quarts seraient juifs et beaucoup sont des Polonais sous tutelle tsariste ${ }^{2}$. Ces immigrés se partagent entre la rive droite (le Marais), où se retrouvent les petits-bourgeois et les ouvriers, et la rive gauche, où habitent les politiques et les universitaires. Dès 1890 se constitue en effet aux limites des $V^{e}$ et XIIIe arrondissements un quartier appelé la "Petite Russie". Selon un rapport de police de 19073, "les Russes orthodoxes habitent de préférence dans le Ve arrondissement, vers la rue Claude-Bernard et le boulevard Saint-Marcel. La rue Flatters, qui fut percée et construite il y a une trentaine d'années, fut dès l'origine habitée par des étudiants russes. Elle est restée le centre de la colonie qui, aujourd'hui, rayonne sur le quartier du Jardin des Plantes, sur le XIII arrondissement et même, depuis trois ou quatre ans, sur le XIVE arrondissement." Fernand Rivoire décrit ainsi ce quartier : "Le principal noyau se trouve dans les rues Pascal, de la Glacière, Berthollet et Broca. Là, on trouve des restaurants russes, des blanchisseuses, des tailleurs et des horlogers, dont les enseignes sont écrites en russe et ornées de rubans jaunes et noirs peints sans délicatesse4." Beaucoup d'étudiants et d'étudiantes russes en médecine y habitent dans des hôtels ou pensions. On y trouve des restaurants où l'on mange pour 9 sous, seulement à midi.

Les étudiants russes logés dans la "Petite Russie" des Gobelins bénéficient de nombreux services. Un rapport de police de 1907 cite les œuvres ouvertes aux Russes dans divers arrondissements de la capitale : le Bureau du travail chargé de venir en aide aux émigrés russes, 63, avenue des Gobelins $\left(X I I I^{e}\right)$, le Comité de bienfaisance de la rue Rodier (IX) $)$, le Comité d'assistance par le travail de la rue Oberkampf $\left(\mathrm{XI}^{\mathrm{e}}\right)$, la Table d'hôte des émigrés, 53, rue de la Glacière, fondée par le Bureau du travail. De nombreux restaurants sont cités dans le quartier russe ou le Quartier latin :15, rue des Écouffes (IVe, métro Saint-Paul), 3, rue de Valence (Ve arrondissement), 9, rue Michel-Peter (XIII', près des Gobelins), 32, rue de l'Arbalète, 55, rue Saint-Jacques, et celui du 11, rue Vauquelin, "où ils se rencontrent chaque jour et où circulent les nouvelles, les mots d'ordre intéressant la colonie".

La police donne aussi une liste des bibliothèques russes, "où sont reçus les journaux du pays russe et qui sont pourvues d'une littérature spéciale". La principale est la bibliothèque Tourgueniev, "sorte de petit État neutre au milieu des divers partis politiques russes qui ont établi leur quartier général à Paris", bibliothèque qui a souvent changé d'adresse : d'abord installée rue Victor-Cousin, elle fut par la suite transférée au 7, rue Berthollet, avant de s'établir au 328, rue Saint-Jacques, puis au 61, avenue des Gobelins, et au 20, rue de la Glacière, avant son installation en décembre 1913 au 9 de la rue du Val-de-Grâce. Elle occupe aujourd'hui un immeuble discret au 11 de la rue de Valence, dans l'ancien quartier russe.

1. Voir, par exemple, “Oiseaux de passage : la colonie russe de Paris”, in L'lllustration, 26 mars 1904, n 3187, p. 204

2. Nancy Green, Les Travailleurs immigrés juifs à la Belle Époque. Le Pletzl de Paris, Paris, Fayard, 1985.

3. Archives nationales, F/7/12894. 4. Fernand Divoire, op. cit. 5. Archives nationales, F/7/12896. 
titulaires d'un doctorat français ${ }^{15}$. C'est pour empêcher les docteurs en médecine reçus à Paris d'exercer leur métier en France que sont institués les diplômes d'université. Le résultat est que, à la veille de la Grande Guerre, le nombre de médecins étrangers exerçant à Paris est peu élevé : en 1911, on ne compte que 541 médecins étrangers installés en France, soit 2,6 \% des praticiens en exercice, dont 355 établis dans la Seine (8,2 \% des médecins de ce département). Cette proportion n'est que de $3 \%$ en 1929 (11\% dans le département de la Seine). Les femmes docteurs ne sont pas mieux traitées : en 1920, la liste des docteurs établis dans le département de la Seine permet de recenser 103 femmes, en majorité françaises et docteurs de la faculté de Paris, sur 4434 docteurs, soit à peine $2,3 \%^{16}$.

La grande majorité des anciens étudiants de Paris repartent dans leur pays, où le diplôme français est souvent coté, notamment dans les pays en développement de l'Est européen et d'Amérique latine : il leur permet souvent d'accéder à des fonctions importantes. Mais leur séjour en France a d'autres conséquences : les étudiants de retour dans leur pays diffusent la langue et la culture françaises, ainsi que la consommation des produits et des modes de vie français, ils véhiculent des idées, philosophiques ou politiques, mais aussi scientifiques et pédagogiques, voire diffusent les valeurs des Lumières, de la démocratie et de la laïcité. Ces retombées mériteraient une étude dans les pays qui envoient en France le plus d'étudiants, qu'il s'agisse de la Russie d'avant 1917, de la Roumanie, de l'Égypte ou de la Turquie, mais elle est difficile à mener en raison du petit nombre de personnes concernées.

Toutefois, nombre de ces anciens élèves des facultés françaises ont mené de brillantes carrières politiques ou scientifiques dans leur pays d'origine ${ }^{17}$. Les réformes de l'enseignement dans leur pays sont souvent inspirées par ce transfert d'idées et d'informations. 